\title{
Epilepsy with Multifocal Spike Discharge in Patients with Severe Motor and Intellectual Disabilities
}

Tomoyuki Takano ${ }^{1 *}$, Keiko Okumura1 ${ }^{1}$, Shosaku Yamazaki ${ }^{1}$, Katsuhiko Ogawa ${ }^{2}$, Yuki Harada ${ }^{3}$, Junko Yorifuji ${ }^{1}$, Rima Ando $^{2}$ and Anri Hayashi $^{4}$

${ }^{1}$ Department of Pediatrics, Biwako-Gakuen Medical and Welfare Center of Yasu, 978-2 Kitazakura, Yasu-Shi, Shiga-Ken, Japan

${ }^{2}$ Department of Internal Medicine, Biwako-Gakuen Medical and Welfare Center of Yasu, 978-2 Kitazakura, Yasu-Shi, Shiga-Ken, Japan

${ }^{3}$ Department of Orthopedics, Biwako-Gakuen Medical and Welfare Center of Yasu, 978-2 Kitazakura, Yasu-shi, Shiga-Ken, Japan

${ }^{4}$ Department of Pediatrics, Shiga Medical Center for Children, 5-7-30 Moriyama, Moriyama-Shi, Shiga-Ken, Japan

\begin{abstract}
Purpose: To characterize the epilepsy in patients with severe motor and intellectual disabilities (SMID), the relationship between the severity of disabilities and the characteristics of epilepsy was examined in 130 children and adults hospitalized in our residential facility.

Methods: Ohshima's classification was used to evaluate the severity of motor and intellectual disabilities. All the following clinical data were collected from the medical records: sex, age, etiology, and clinical features of epilepsy. The participants were divided into the profound SMID and less-profound SMID groups according to the Ohshima's classification score.

Results: The main findings were as follows: (1) the severity of motor and intellectual disabilities was higher in the epilepsy-positive SMID patients than in the epilepsy-negative SMID patients ( $p=0.001) ;(2)$ an etiological analysis indicated the relatively higher incidence of neonatal hyperbilirubinemia $(p=0.005)$ and traumatic brain injury $(p=0.027)$ in the epilepsy-negative and the epilepsy-positive patients, respectively; (3) the mean number of antiepileptic drugs administered in the profound SMID group was higher than that in the less-profound SMID group $(p=0.0008)$; and $(4)$ an EEG analysis revealed the higher detection rate of multifocal spike discharge in the profound SMID group than that in the less-profound SMID group $(p=0.031)$.
\end{abstract}

Conclusion: While the refractory mechanism is unclear, multifocal epileptogenesis was thought to be one of the most characteristic findings in patients with SMID and may be closely related to intractability of epilepsy.

Keywords: Severe motor and intellectual disabilities; Epilepsy; Multifocal spike discharge; Multifocal epileptogenesis

\section{Introduction}

The term of "severe motor and intellectual disabilities (SMID)" has been used to describe a heterogeneous group of disorders that include both severe physical disabilities and profound mental retardation, a concept that is based on social welfare terminology (not medical terminology) and which was developed originally in Japan [1]. While Western countries may lack an identical concept equivalent to SMID, the term "severe or profound intellectual and motor disabilities (SPIMD)" seems to be established and is frequently used to describe patients with a combination of a severe or profound intellectual disability (ID) (i.e., estimated intelligence quotient $<35$ ) and profound motor disability (i.e., inability to move independently) [2]. Patients with SPIMD frequently experience health problems, such as dysphagia, epilepsy, spasticity, respiratory dysfunction, urination disorders, and hearing and visual impairment $[3,4]$. The estimated life expectancy of people with severe intellectual and motor disabilities is lower than that of people with a milder intellectual disability and the general population [5-7]. Strong evidence also shows that the physical inactivity, which seems to be a feature of SMID patients, increases the risk of many adverse health conditions, including major noncommunicable diseases such as coronary heart disease, type 2 diabetes, and breast and colon cancers, and shortens life expectancy [8].

Epilepsy is one of the most common comorbidities in patients with SMID. Its approximate prevalence is $70-80 \%$, and with insufficient seizure control $[2,3]$. Some studies have reported on epilepsy in patients with SMID, emphasizing the importance of early seizure control, indicating the important role of electroencephalogram (EEG) findings, and identifying poor prognostic factors for seizure control $[9,10]$. However, the pathogenetic mechanisms underlying intractable epilepsy among SMID patients have yet to be clarified. In the present study, to characterize the epilepsy in patients with SMID, we examined the relationship between the severity of disabilities and the clinical features of epilepsy in 130 children and adults hospitalized in our residential facility. Section numbers of Ohshima's classification was used to grade the severity of disabilities [11].

\section{Methods}

\section{Study participants}

Our residential facility hospital Biwako-gakuen was established in 1963 for the medical and welfare treatment of children with mental and physical disabilities. Because of their profound disabilities, most of the residential patients need to be hospitalized for their entire life. At the time of this study, the hospitalization period of residential patients ranged from 1 year to 56 years. The study population included 130

*Corresponding author: Tomoyuki Takano, Department of Pediatrics, Biwako-Gakuen Medical and Welfare Center of Yasu, 978-2 Kitazakura, Yasu-Shi, Shiga-Ken 520-2321, Japan, Tel: 8177587 1144; E-mail: tmyktkn@belle.shiga-med.ac.jp

Received August 27, 2018; Accepted October 11, 2018; Published October 15, 2018

Citation: Takano T, Okumura K, Yamazaki S, Ogawa K, Harada Y, et al. (2018) Epilepsy with Multifocal Spike Discharge in Patients with Severe Motor and Intellectual Disabilities. J Neurol Disord 6: 391. doi:10.4172/2329-6895.1000391

Copyright: () 2018 Takano T, et al. This is an open-access article distributed under the terms of the Creative Commons Attribution License, which permits unrestricted use, distribution, and reproduction in any medium, provided the original author and source are credited. 
mentally and physically disabled children and adults, all of whom were residential clients hospitalized at this facility. All examinations and treatments administered to the patients are regular practice based on medical necessity without any intervention. The present retrospective study analyzed the clinical history of 130 patients and was approved by the ethics committee of the Biwako-gakuen Medical and Welfare Center of Yasu on October 1, 2017.

\section{Procedure}

Section numbers of Ohshima's classification, which classifies the level of each motor and intellectual disability by combining them into 25 different divisions (with 1 and 25 indicating the highest and lowest degrees of disability, respectively), was used to evaluate the severity of motor and intellectual disabilities, as scoring of degree of SMID (Table 1) [11]. SMID was defined as a classification of 1-9 under this system, with classes 1-4 defined as extreme SMID. These definitions are widely used to classify motor and intellectual disabilities in children and adults in Japan. All clinical data were collected between October 2017 and April 2018, and the following items were investigated from the medical records: sex, age, etiology, Ohshima's classification number, and the clinical features of epilepsy. To analyze the relationship between the severity of disabilities and the characteristics of epilepsy in patients with mental and physical disabilities, all participants were included as SMID in this study and divided into the profound SMID (score $\leq 4)$ and less-profound SMID (score $\geq 5$ ) groups according to the Ohshima's classification number.

The terminology for seizures and epilepsy and epilepsy classification were based on the most recent ILAE commission reports and comments

\begin{tabular}{|c|c|c|c|c|c|}
\hline \multicolumn{7}{|c|}{ Motor Function Level } \\
\hline IQ level & Run & Walk & $\begin{array}{c}\text { Walk with } \\
\text { assistance }\end{array}$ & Sit & Bed ridden \\
\hline $70-100$ & 21 & 22 & 23 & 24 & 25 \\
\hline $50-69$ & 20 & 13 & 14 & 15 & 16 \\
\hline $35-49$ & 19 & 12 & 7 & 8 & 9 \\
\hline $20-34$ & 18 & 11 & 6 & 3 & 4 \\
\hline $0-19$ & 17 & 10 & 5 & 2 & 1 \\
\hline
\end{tabular}

Table 1: The Ohshima's classification score table. This table shows the 25 different divisions defined by the intelligence quotient (IQ) and motor function level.
$[12,13]$, including the guideline from the ILAE website (www. epilepsydiagnosis.org). The etiology of mental and physical disabilities was classified into 10 items based on the etiological classification of epilepsy, as follows: chromosomal abnormalities, gene abnormalities, malformation of cortical development, hypoxic-ischemic brain injury, traumatic brain injury, brain tumors, porencephalic cyst, metabolic disease, infectious disease, and unknown etiology. Epilepsy types were divided into four groups, as follows: (1) focal epilepsy, (2) generalized epilepsy, (3) combined generalized and focal epilepsy, and (4) unknown type. As epilepsy syndromes often transform with age in patients with a long clinical course, patients were roughly divided into the two groups of syndromic and non-syndromic epilepsy according to the presence of epileptic syndromes at the time of this study. And non-syndromic epilepsies were further classified according to the epilepsy types. The EEG recordings used the 10-20 international system of electrode placement with referential and bipolar montage. Activation procedures with photic stimulation and hyperventilation were routinely performed if the patient was able to cooperate. EEG findings were classified into five groups according to the interictal paroxysmal discharges, as follows: (1) No spike discharge, (2) Focal spike discharge, (3) Hemispheric spike discharge, (4) Multifocal spike discharge (defined as the presence of more than two independent spike foci), and (5) Generalized spike discharge. Statistical significance was determined using Welch's $t$-test and the $\chi^{2}$ test, and $\mathrm{p}$ values of $<0.05$ were considered statistically significant.

\section{Results}

\section{Comparison of SMID patients with and without epilepsy}

The total number of SMID patients in this study was 130 (male/ female $=73 / 57$ ). The mean age of the patients was 51.1 years (range, 11 to 85 years) and the mean Ohshima's classification score was 4.49 (range, 1 to 18 ). In the epilepsy-negative 63 patients (48.5\%; male/ female $=32 / 31$ ), the mean age was 56.4 years (range, 15 to 85 years). In the epilepsy-positive 67 patients $(51.5 \%$; male/female $=41 / 26)$, the mean age was 46.1 years (range, 11 to 70 years). The mean Ohshima's classification scores of the epilepsy-negative and epilepsy-positive groups were 5.79 and 3.26 , respectively $(\mathrm{p}=0.001)$. The number of patients with each Ohshima's classification score (1-18) is shown in

\begin{tabular}{|c|c|c|c|c|}
\hline Variables & Total & Epilepsy (-) & Epilepsy (+) & $p$-value \\
\hline Number of patients & 130 & $63(48.5)$ & $67(51.5)$ & -- \\
\hline Male/Female & $73 / 75$ & $32 / 31$ & $41 / 26$ & -- \\
\hline Mean age, years (SD) & 51.1 (13.9); range $11-85$ & 56.4 (12.3); range $15-85$ & 46.1 (13.5); range $11-70$ & -- \\
\hline $\begin{array}{l}\text { Mean score of Ohshima } \\
\text { classification (SD) }\end{array}$ & 4.49 (4.55); range $1-18$ & 5.79 (5.11); range 1-18 & 3.26 (5.11); range $1-17$ & 0.001 \\
\hline \multicolumn{5}{|l|}{ Etiology (\%) } \\
\hline Chromosomal abnormalities & $4(3.1)$ & $1(1.6)$ & $3(4.5)$ & -- \\
\hline Gene abnormalities & $13(10.0)$ & $6(9.5)$ & $7(10.4)$ & -- \\
\hline $\begin{array}{c}\text { Malformation of cortical } \\
\text { development }\end{array}$ & $9(6.9)$ & $4(6.3)$ & $5(7.5)$ & -- \\
\hline Hypoxic -Ischemic brain injury & $40(30.8)$ & $22(34.9)$ & $18(26.9)$ & -- \\
\hline Traumatic brain injury & $5(3.8)$ & $0(0)$ & $5(7.5)$ & 0.027 \\
\hline Brain tumours & $1(0.7)$ & $1(1.6)$ & $0(0)$ & -- \\
\hline Porencephalic cyst & $1(0.7)$ & $0(0)$ & $1(1.5)$ & -- \\
\hline Metabolic disease & $7(5.4)$ & $7(11.1)^{*}$ & $0(0)$ & 0.005 \\
\hline Infectious disease & $13(10.0)$ & $4(6.3)$ & $9(13.4)$ & -- \\
\hline Unknown & $37(28.5)$ & $18(28.6)$ & $19(28.4)$ & -- \\
\hline
\end{tabular}

Table 2: The age, Ohshima's classification score and etiological classification of the Epilepsy (-) and Epilepsy (+) patients. 
Citation: Takano T, Okumura K, Yamazaki S, Ogawa K, Harada Y, et al. (2018) Epilepsy with Multifocal Spike Discharge in Patients with Severe Motor and Intellectual Disabilities. J Neurol Disord 6: 391. doi:10.4172/2329-6895.1000391

Page 3 of 6

\begin{tabular}{|c|c|c|c|c|}
\hline Variables & Total & $\begin{array}{l}\text { Profound SMID: Ohshima's } \\
\text { classification score } \leqq 4\end{array}$ & $\begin{array}{l}\text { Less-profound SMID: Ohshima's } \\
\text { classification score } \geqq 5\end{array}$ & $p$-value \\
\hline Number of patients (\%) & 67 & $48(71.6)$ & $19(28.4)$ & -- \\
\hline Male/Female & $41 / 26$ & $28 / 20$ & $13 / 16$ & -- \\
\hline Mean age, years (SD) & 46.1 (13.5); range $11-70$ & 43.2 (13.7); range $11-70$ & 53.6 (10.1); range $35-67$ & 0.001 \\
\hline $\begin{array}{l}\text { Mean score of Ohshima's } \\
\text { classification (SD) }\end{array}$ & 3.26 (3.57); range $1-17$ & $1.47(0.65)$; range $1-4$ & 7.78 (3.95); range $5-17$ & $1.802 \times 10^{-6}$ \\
\hline \multicolumn{5}{|l|}{ Seizure onset (\%) } \\
\hline$<1$ year & $28(41.8)$ & $20(41.7)$ & $8(42.1)$ & \multirow{5}{*}{--} \\
\hline $1-5$ & $19(28.4)$ & $12(25.0)$ & $7(36.8)$ & \\
\hline $6-10$ & $11(46.4)$ & $9(18.8)$ & $2(10.5)$ & \\
\hline $11-15$ & $4(6.0)$ & $4(8.3)$ & $0(0)$ & \\
\hline 16 & $5(7.5)$ & $3(6.3)$ & $2(10.5)$ & \\
\hline $\begin{array}{l}\text { Mean age of seizure onset, years } \\
\text { (SD) }\end{array}$ & $5.3(9.4)$; range $0-56$ & 5.3 (8.9); range $0-56$ & 5.3 (10.7); range $0-46$ & \\
\hline \multicolumn{5}{|l|}{ Epilepsy types (\%) } \\
\hline Focal & $24(35.8)$ & $15(31.3)$ & $9(47.4)$ & \multirow{3}{*}{--} \\
\hline Generalized & $14(20.9)$ & $10(20.8)$ & $4(21.1)$ & \\
\hline Combined generalized and focal & $29(43.3)$ & $23(47.9)$ & $6(31.6)$ & \\
\hline \multicolumn{5}{|l|}{ Epilepsy syndromes (\%) } \\
\hline Syndromic epilepsy & $12(17.9)$ & & & \multirow{6}{*}{--} \\
\hline LGS & $12(17.9)$ & $9(18.8)$ & $3(15.8)$ & \\
\hline Non-syndromic epilepsy & $55(82.1)$ & & & \\
\hline Focal & $24(35.8)$ & $15(31.3)$ & $9(47.4)$ & \\
\hline Generalized & $14(20.9)$ & $10(20.8)$ & $4(21.1)$ & \\
\hline Combined generalized and focal & $17(25.4)$ & $14(29.2)$ & $3(15.8)$ & \\
\hline \multicolumn{5}{|l|}{ Seizure types (\%) } \\
\hline $\begin{array}{l}\text { Focal onset impaired awareness } \\
\text { seizures }\end{array}$ & $52(40.3)$ & $36(37.5)$ & $16(48.5)$ & \multirow{4}{*}{--} \\
\hline Generalized onset motor seizures & $70(54.3)$ & $55(57.3)$ & $15(45.5)$ & \\
\hline Generalized onset non-motor seizures & $7(5.4)$ & $5(5.2)$ & $2(6.1)$ & \\
\hline Total & 129 & 96 & 33 & \\
\hline \multicolumn{5}{|l|}{ Seizure frequency (\%) } \\
\hline Day & $2(3.0)$ & $2(4.2)$ & $0(0)$ & \multirow{5}{*}{--} \\
\hline Week & $12(17.9)$ & $10(20.8)$ & $2(10.5)$ & \\
\hline Month & $14(20.9)$ & $10(20.8)$ & $4(21.1)$ & \\
\hline Year & $20(29.9)$ & $15(31.3)$ & $5(26.3)$ & \\
\hline Controlled ( $>3$ years) & $19(28.4)$ & $11(22.9)$ & $8(42.1)$ & \\
\hline \multicolumn{5}{|l|}{ Number of AEDs (\%) } \\
\hline 1 & $21(31.3)$ & $11(22.9)$ & $10(52.6)$ & 0.018 \\
\hline 2 & $16(23.9)$ & $11(22.9)$ & $5(26.3)$ & -- \\
\hline 3 & $13(19.4)$ & $10(20.8)$ & $3(15.8)$ & -- \\
\hline 4 & $11(16.4)$ & $10(20.8)$ & $1(5.3)$ & -- \\
\hline 5 & $6(9.0)$ & $6(12.5)$ & 0() & -- \\
\hline Mean number of AEDs (SD) & $2.4(1.3)$; range $1-5$ & $2.7(1.3)$; range $1-5$ & $1.7(0.9)$; range $1-3$ & 0.0008 \\
\hline \multicolumn{5}{|c|}{ EEG paroxysm (interictal discharges) (\%) } \\
\hline No spike discharge & $26(38.8)$ & $16(33.3)$ & $10(52.6)$ & -- \\
\hline Focal spike discharge & $24(35.8)$ & $18(37.5)$ & $6(31.6)$ & -- \\
\hline Hemispheric spike discharge & $2(3.0)$ & $1(2.1)$ & $1(5.3)$ & -- \\
\hline Multifocal spike discharge & $10(14.9)$ & $10(20.8)$ & $0(0)$ & 0.031 \\
\hline Generalized spike discharge & $5(7.5)$ & $3(6.3)$ & $2(10.5)$ & -- \\
\hline
\end{tabular}

Table 3: The characteristics of epilepsy in patients with profound and less-profound SMID.

Figure 1. An etiological classification analysis revealed that hypoxicischemic brain injury was the most frequent etiology (30.8\%), while brain tumors and porencephalic cyst were the rarest (each $0.7 \%$ ). On comparing the epilepsy-negative and epilepsy-positive groups, the incidence of metabolic disease $(n=7)$ was significantly higher in the epilepsy-negative group $(\mathrm{p}=0.005)$, while that of traumatic brain injury $(n=5)$ was significantly higher in the epilepsy-positive group ( $\mathrm{p}=0.027$ ). All seven patients categorized as having metabolic disease in the epilepsy-negative group were patients with neonatal hyperbilirubinemia (Table 2).

\section{Characteristics of epilepsy in the profound and less-pro- found SMID groups}

In the 67 epilepsy-positive patients, profound SMID was 48 patients $(71.6 \%$; male/female $=28 / 20)$, while less-profound SMID was 19 patients $(28.4 \%$; male/female $=13 / 6)$. The mean age of the 


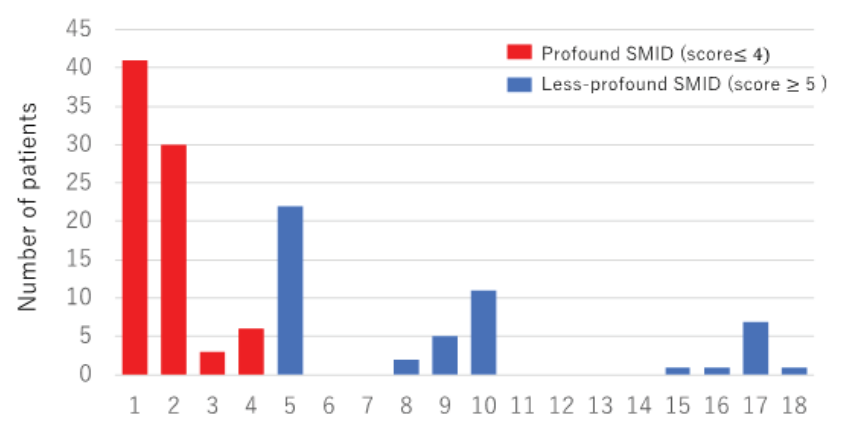

Ohshima's classification score

Figure 1: Numbers of patients with each Ohshima's classification score (118). Profound and less-profound SMID indicate patients with scores of $\leq 4$ and $\geq 5$ respectively.

patients was 43.2 years (range: $11-70$ years) and 53.6 years (range: 35-67 years) in the profound and the less-profound SMID groups, respectively $(\mathrm{p}=0.001)$. The mean Ohshima's classification score was 1.47 (range: 1-4) and 7.78 (range: 5-17) in the profound and the lessprofound SMID groups, respectively. The age of seizure onset ranged widely from 0 days to 56 years, but an onset at $<1$ year of age was the most frequently reported, that is, $41.7 \%$ and $42.1 \%$ of the patients in the profound and the less-profound SMID groups, respectively (not significant). The mean age at seizure onset was 5.3 years in both groups. The predominant epilepsy types were combined generalized and focal epilepsy in the profound SMID group (47.9\%) and focal epilepsy in the less-profound SMID group (47.4\%). Most epilepsy patients with SMID had non-syndromic epilepsy (82.1\%); few showed specific clinical features of epileptic syndrome, which was restricted to Lennox-Gastaut syndrome (LGS) (17.9\%). The incidence of LGS in the profound (18.8\%) and less-profound (15.8\%) SMID cases did not differ to a statistically significant extent. More than $90 \%$ of patients showed focal-onset impaired awareness seizures and generalized-onset motor seizures. The seizure frequency ranged from daily to yearly, and 11 (22.9\%) patients in the profound SMID group and 8 patients $(42.1 \%)$ in the less-profound SMID group showed more than 3 years of seizure control (not significant) (Table 3).

The mean number of antiepileptic drugs administered was 2.7 (range: 1-5) and 1.7 (range: 1-3) in the profound and the less-profound groups, respectively $(\mathrm{p}=0.0008)$. The proportion of monotherapy by antiepileptic drugs in the less-profound SMID group (52.6\%) was significantly higher than that in the profound SMID group (22.9\%) $(\mathrm{p}=0.018)$. An EEG analysis to investigate interictal discharges revealed that the most frequent paroxysm pattern was focal spike discharge in both the profound (37.5\%) and less-profound (31.6\%) groups, and that more than half of the patients in the less-profound SMID group (52.6\%) showed no spike discharge. Multifocal spike discharge was detected in 10 persons $(20.8 \%)$ in the profound SMID group, which was significantly higher than that in the less-profound SMID group $(\mathrm{p}=0.031)$.

\section{Discussion}

The main findings obtained in this study were as follows:

1. The analysis of the Ohshima's classification scores revealed that the severity of motor and intellectual disabilities was higher in the epilepsy-positive SMID patients than that in the epilepsynegative SMID patients $(\mathrm{p}=0.001)$.
2. An etiological analysis of the SMID patients indicated the relatively higher incidence of neonatal hyperbilirubinemia $(\mathrm{p}=0.005)$ and traumatic brain injury $(\mathrm{p}=0.027)$ in the epilepsynegative and the epilepsy-positive patients, respectively.

3. In 67 patients with epilepsy, the mean age of patients in the profound SMID group was younger than that of the lessprofound SMID group $(\mathrm{p}=0.001)$.

4. The mean number of antiepileptic drugs administered in the profound SMID group was higher than that in the less-profound SMID group $(\mathrm{p}=0.0008)$.

5. An EEG analysis revealed the higher detection rate of multifocal spike discharge in the profound SMID group than that in the less-profound SMID group $(\mathrm{p}=0.031)$.

The prevalence of epilepsy in people with intellectual disabilities is significantly greater than that in the general population, with the estimated incidence in individuals with intellectual disability ranging from $18 \%$ to $>60 \%$ [14]. The increasing prevalence of epilepsy with the increasing severity of intellectual disability is well recognized in both children [15] and adults [16]. Individuals with mental retardation are more likely to have epilepsy if they also have cerebral palsy, and those with cerebral palsy are more likely to have epilepsy if they are mentally retarded. To a certain extent, these relationships may reflect a common etiology; however, it is not possible to draw hard conclusions [17]. The risk of epilepsy in different subtypes of cerebral palsy also varies, with the highest risk being reported in individuals with quadriplegia or dystonic cerebral palsy [18]. In patients with spastic hemiplegia, the relative incidence of epilepsy is intermediate, with the lowest frequency being reported in those with preterm-related diplegia or athetoid cerebral palsy [19]. Thus, epilepsy is one of the most frequent complications in patients with motor and intellectual disabilities. In our institution the frequency of epilepsy was $51.5 \%$, and comorbid epilepsy was more frequently recognized in more profoundly disabled patients than in those with milder disability, suggesting that more profound brain dysfunction was associated with increased epileptogenicity.

The common neuropathological processes among the three disorders: epilepsy, intellectual disabilities, and motor dysfunction, have yet to be clarified. Inhibitory interneurons are thought to be the main cellular elements that control hyperexcitability in the brain [20], and interneuron dysfunction can cause pathological hyperexcitability linked to seizure susceptibility or epilepsy [21,22]. Autism is an extremely heterogeneous intellectual disorder and is frequently recognized in epilepsy [23,24]. The prevalence of epilepsy in patients with autism ranges from $5 \%$ to $46 \%$ and is increased in those with greater intellectual disability [25]. Its high merge ratio with epilepsy leads to speculation that these disorders may share common mechanisms. In our review of five genetic syndromes associated with autism, we reported that Fragile X syndrome, Angelman syndrome, and Tuberous sclerosis complex mainly showed the downregulation of the inhibitory neuronal function [26], whereas Neurofibromatosis type 1 primarily showed upregulation in the $\gamma$-aminobutyric acid (GABA)mediated inhibitory system. In Rett syndrome, the GABAergic presynaptic function was mainly downregulated; however, a stronger innervation of the pyramidal neurons by parvalbumin-expressing interneurons was also reported, suggesting the presence of both dysregulation mechanisms in the inhibitory function [26].

Periventricular leukomalacia (PVL) is the most frequent cause of spastic diplegia in cerebral palsy. The movement disorder is attributed to damage to the corticospinal tract, but there is increasing 
evidence of additional cortical dysfunction associated with PVL. A study of the integrity of the corticospinal tract and the cortical inhibitory function using transcranial magnetic stimulation revealed the cortical involvement in the reduced cortical inhibitory function, suggesting impaired functioning of the cortical interneurons involving thalamocortical or cortico-cortical connections in PVL [27]. These findings suggest that interneurons may play a crucial role in all three fundamental disorders of SMID: epilepsy, intellectual disabilities, and motor dysfunction.

Traumatic brain injury is a growing silent and major contributor to increases in the long-term risk for neurological dysfunction, including drug resistance-acquired epilepsy [28]. Brain injuries ranging from mild trauma to severe penetrating injuries are associated with an increased risk of epilepsy. There appears to be a strong correlation between the injury severity and the risk for developing spontaneous seizures [29]. Both clinical and experimental data indicate that the developing brain is more susceptible to post-traumatic epileptogenesis than an adult brain $[30,31]$. Risk factors for developing epilepsy that appear common to multiple acute injury etiologies include intracranial bleeding, disruption of the blood-brain barrier, more severe injury, and early seizures within one week of injury [32]. Recent studies have revealed diverse pathologies for epileptogenesis, including enhanced excitability, activated immune signaling, cell death, and enhanced neurogenesis within a week after injury, suggesting a period of heightened adaptive and maladaptive neuronal plasticity [33]. The relatively high incidence of traumatic brain injury as a cause of epilepsy in cases of profound SMID is suggested to be closely associated to the multidimensional nature of epileptogenesis induced by more profound brain dysfunction.

The clinical characteristics of epilepsy with multifocal interictal EEG spikes presented in our study, findings that were mostly detected in the patients with profound SMID, are thought to be essentially identical to those of "severe epilepsy with multiple independent spike foci (SE-MISF)" reported by Yamatogi and Ohtahara [34]. SE-MISF has the following characteristics:

1. EEG showing multiple independent spike foci (three or more foci in both hemispheres; i.e., at least one in each hemisphere) and diffuse slowing of background activity;

2. Very frequent multiple types of seizures but mainly generalized minor seizures;

3. A frequent association with mental retardation and neurologic abnormalities;

4. Underlying causes of various nonspecific prenatal, perinatal, and postnatal cerebral conditions;

5. Poor prognoses for seizures and psychomotor development.

In the study of EEG in SMID patients, it has been reported that the rate of Ohshima's classification 1 was highest in SE-MISF [9], and multifocal spikes or diffuse spike and waves were suggested to be one of the statistically significant poor prognostic factors in SMID [10]. While the exact role of multiple independent spike foci in the epileptic network has not been clarified, our present results suggest that multifocal spike discharge may be induced by multiple brain lesions inducing more profound motor and intellectual disabilities and is closely associated with multifocal epileptogenesis, as reflected by refractory epilepsy in SMID patients.

\section{Conclusion}

SMID is frequently accompanied by epilepsy, and the intractability of epilepsy in such cases is associated with the severity of motor and intellectual disabilities. The epileptogenesis in these cases may have a multidimensional nature, including enhanced excitability, activated immune signaling, cell death, enhanced neurogenesis, and interneuron dysfunction. One of the most characteristic EEG findings in our study was the higher detection rate of multifocal spike discharge in the more profoundly disabled patients. While the refractory mechanism is unclear, multifocal epileptogenesis was thought to be one of the most characteristic findings in patients with SMID and may be closely related to intractability of epilepsy.

\section{Acknowledgements}

This work was supported by a KAKENHI Grant-in-Aid for Scientific Research (C; No. 15K09671).

\section{References}

1. Arima M (1996) Future perspective in the study of developmental disabilities Hattatsu Shogai Kenkyu, Tokyo 17: 264-266.

2. van Timmeren EA, Van der Putten AAJ, Van Schrojenstein Lantman-de Valk HMJ, Van der Schans CP, Waninge A (2016) Prevalence of reported physical health problems in people with severe or profound intellectual and motor disabilities: A cross-sectional study of medical records and care plans. J Intellect Disabil Res 60: 1109-1118.

3. Van Timmeren EA, Van der Schans CP, Van der Putten AAJ, Krijnen WP, Steenbergen HA, et al. (2017) Physical health issues in adults with severe or profound intellectual and motor disabilities: A systematic review of crosssectional studies. J Intellect Disabil Res 61: 39-49.

4. Van Timmeren EA, Waninge A, Van Schrojenstein Lantman-de Valk HMJ, Van de Putten AAJ, Van der Schans CP (2017) Patterns of multimorbidity in people with severe or profound intellectual and motor disabilities. Res Dev Disabil 67: 28-33.

5. Patja K, Livanainen M, Vesala H, Oksanen H, Ruoppila I (2000) Life expectancy of people with intellectual disability: a 35 -year follow-up study. J Intellect Disabil Res 44: 591-599.

6. Coppus AMW (2013) People with intellectual disability: What do we know about adulthood and life expectancy? Dev Disabil Res Rev 18: 6-16.

7. Heslop P, Blair PS, Fleming P, Hoghton M, Marriott A, et al. (2014) The confidential inquiry into premature deaths of people with intellectual disabilities in the UK: A population-based study. Lancet 383: 889-895.

8. Lee IM, Shiroma EJ, Lobelo F, Puska P, Blair SN, et al. (2012) Effect of physical inactivity on major non-communicable diseases worldwide: an analysis of burden of disease and life expectancy. Lancet 383: 219-229.

9. Matsumoto A, Miyazaki S, Hayakawa C, Komori T, Nakamura M (2007) Epilepsy in severe motor and intellectual disabilities syndrome (SMIDS): A clinical and electroencephalographic study of epileptic syndromes. Epilepsy Res 77: 120-127.

10. Matsumoto A, Miyazaki S, Hayakawa C, Komori T, Nakamura M, et al. (2009) Prognostic factors for epileptic seizures in severe motor and intellectual disabilities syndrome (SMIDS): A clinical and electroencephalographic study. Epilepsy Res 86: 175-182.

11. Ohshima K (1971) Basic problem of severely, mentally, and physically disabled children. Koshu Eisei, Tokyo . 648-655.

12. Scheffer IE, Berkovic S, Capovilla G, Connolly MB, French J, et al. (2017) ILAE classification of the epilepsies: position paper of the ILAE commission for classification and terminology. Epilepsia 58: 512-521.

13. Fisher RS, Cross JH, French JA, Higurashi N, Hirsch E, et al. (2017) Operationa classification of seizure types by the International League Against Epilepsy: position paper of the ILAE commission for classification and terminology. Epilepsia 58: 522-530.

14. Kerr MP, Smith ML (2008) Learning disorders. In J. Engel Jr., T. A. Pedley (Eds.), Epilepsy: A comprehensive textbook, Wolters Kluwer/Lippincott Williams \& Wilkins, Philadelphia, USA. pp: 2093-2103.

15. Airaksinen EM, Matilainen R, Mononen T, Mustonen K, Partanen J, et al (2000) A population-based study on epilepsy in mentally retarded children. Epilepsia 41: 1214-1220.

16. Amano K, Takamatsu J, Ogata A, Miyazaki C, Kaneyama H, et al. (2000) 
Citation: Takano T, Okumura K, Yamazaki S, Ogawa K, Harada Y, et al. (2018) Epilepsy with Multifocal Spike Discharge in Patients with Severe Motor and Intellectual Disabilities. J Neurol Disord 6: 391. doi:10.4172/2329-6895.1000391

Characteristics of epilepsy in severe mentally retarded individuals. Psychiatry Clin Neurosci 54: 17-22.

17. Stephenson JBP (2008) Cerebral palsy. In: J. Engel Jr., T. A. Pedley (Eds.), Epilepsy: A comprehensive textbook (pp. 2631-2636). Philadelphia: Wolters Kluwer/ Lippincott Williams \& Wilkins.

18. Edebol-Tysk K (1989) Epidemiology of spastic tetraplegic cerebral palsy in Sweden. I. Impairment and disabilities. Neuropediatrics 20: 41-45.

19. Uvebrant P (1988) Hemiplegic cerebral palsy: Aetiology and outcome. Acta Paediatrica Scandinavia 345: 1-100.

20. Dichter MA, Ayala GF (1987) Cellular mechanisms of epilepsy: A status report. Science 237: 157-164.

21. Takano T (2011) Seizure susceptibility in polymicrogyria: Clinical and experimental approaches. Epilepsy Res 96: 1-10.

22. Takano T, Matsui K (2014) Increased expression of GAP43 in interneurons in a rat model of experimental polymicrogyria. J Child Neurol 30: 716-728.

23. Tuchman R, Rapin I (2002) Epilepsy in autism. Lancet Neurol 1: 352-358.

24. Tuchman R, Cuccaro M, Alessandri M (2010) Autism and epilepsy: Historical perspective. Brain Dev 32: 709-718.

25. Spence SJ, Schneider MT (2009) The role of epilepsy and epileptiform EEGs in autism spectrum disorders. Pediatr Res 65: 599-606.

26. Takano T (2014) Interneuron dysfunction in syndromic autism: Recent advances. Dev Neurosci 37: 467-475.
27. Vry J, Linder-Lucht M, Berweck S, Bonati U, Hodapp M, et al. (2008) Altered cortical inhibitory function in children with spastic diplegia: A TMS study. Exp Brain Res 186: 611-618.

28. Gupta PK, Sayed N, Ding K, Agostini MA, Van Ness PC, et al. (2014). Subtypes of post-traumatic epilepsy: clinical, electrophysiological, and imaging features. J Neurotrauma 31: 1439-1443.

29. Yeh CC, Chen TL, Hu CJ, Chiu WT, Liao CC (2013) Risk of epilepsy after traumatic brain injury: a retrospective population-based cohort study. J Neuro Neurosurg Psychiatry 84: 441-445.

30. Keret A, Bennett-Back O, Rosenthal G, Gilboa T, Shweiki M, et al. (2017) Posttraumatic epilepsy: long-term follow-up of children with mild traumatic brain injury. J Neurosurg Pediatr 20: 64-70.

31. Semple BD, O'Brien TJ, Gimlin K, Wright DK, Kim SE, et al. (2017). Interleukin-1 Receptor in Seizure Susceptibility after Traumatic Injury to the Pediatric Brain J Neurosci 37: 7864-7877.

32. Klein P, Dingledine R, Aronica E, Bernard C, Blumcke I, et al. (2017) Commonalities in epileptogenic processes from different acute brain insult: Do they translate? Epilepsia 59: 37-66.

33. Neuberger EJ, Gupta A, Subramanian D, Korgaonkar AA, Santhakuma $V$ (2017) Converging early responses to brain injury pave the road to epileptogenesis. J Neurol Res 1-10.

34. Yamatogi Y, Ohtahara S (2003) Severe epilepsy with multiple independent spike foci. J Clin Neurophysiol 20: 442-448. 